\title{
Berufliche Kompetenz und Professionalisierung - Testverfahren und Ergebnisse im Spiegelbild ihrer Accountability
}

\author{
Esther Winther • Manfred Prenzel
}

\section{Vorbemerkungen}

Spätestens seit der Etablierung der Schulleistungsvergleiche beginnend mit dem IEA Six Subject Survey (u. a. Walker et al. 1976) in den 1970er Jahren stellt sich die Frage nach der anwendungsorientierten Nutzung und Belastbarkeit von Prüfungs- bzw. Assessmentverfahren. Mit der Implementation der National Vocational Qualifications (NVQ) und General National Vocational Qualifications (GNVQ) im Vereinigten Königreich sowie mit deren Übersetzung in einen europäischen und nationalen Qualifikationsrahmen werden diese Fragen auch für den Bereich der beruflichen Bildung tragend. Die Forschungsdesiderate eines kompetenzbasierten Prüfens zeigen sich vor dem Hintergrund der politischen Entwicklungen als besonderes drängend, aber gleichzeitig auch als steuerungsanfällig und zu wenig auf den zu erwartenden Output von Bildung bezogen:

A curious aspect of competence-based reform, [...] is that, although the reformers' ambitions are very wide, their focus has been very narrow. They would like to see major changes in the whole institutional context of vocational training and education but they have themselves treated the approach as essentially a technical affair. (Wolf 1995, S. 131)

Für die deutsche Diskussion kommt erschwerend hinzu, dass eine systematische Erfassung der Leistungsfähigkeiten von Auszubildenden sowie der institutionellen Ausbildungsprogramme noch aussteht. In den vergangenen Jahren wurden - ausgehend von den Überlegungen eines Berufsbildungs-PISA (vgl. Baethge et al. 2006) - die Forschungsfra-

(C) Springer Fachmedien Wiesbaden 2013

Prof. Dr. E. Winther $(\bowtie)$

Universität Paderborn, Warburger Straße 100,

33098 Paderborn, Deutschland

E-Mail: esther.winther@wiwi.upb.de

Prof. Dr. M. Prenzel

TU München, Arcisstr. 21, 80333 München, Deutschland

E-Mail: manfred.prenzel@tum.de 
gen vorranging auf die Instrumentenentwicklung bezogen und unter der Perspektive einer theoretischen Modellierung beruflicher Kompetenz gebündelt (vgl. Seeber et al. 2010). Der Frage nach der Accountability im Sinne einer umfassenden Legitimationsfähigkeit der Prüfungen bzw. kompetenzbasierten Assementverfahren und damit nach der Rechenschaftslegung der Prüfungs- bzw. Assessmentpraxis wurde bislang wenig Beachtung geschenkt (vgl. hierzu Beiträge in Ryan und Shepard 2008; Wilson 2004). Forschungsdesiderate in diesem Zusammenhang betreffen zum einen die Frage, in welchem Umfang und auf Basis welchen Abstraktionsgrades sich die erzielten Testergebnisse dafür nutzen lassen, die spezifischen Ziele der beruflichen Bildungsgänge zu evaluieren und gegebenenfalls zu modifizieren. Zum anderen bestehen in Hinblick auf die theoretischen Ausarbeitungen wie empirischen Modellierungen Unsicherheiten darüber, wie sich Leistungszuwächse und Ergebnisse beruflicher Professionalisierung analysieren und interpretieren lassen. Die Beantwortung dieser Fragen setzt eine Kenntnis davon voraus, welche Ziele mit dem Assessment verfolgt werden sollen. So werden in der beruflichen Bildung beispielsweise Unterscheidungen zwischen den verschiedenen Lernorten getroffen: Ist das Assessment auf einen konkreten Arbeitsplatz bezogen oder soll es die generellen, institutionellen Leistungszuwächse abbilden? Sind Inhalte und Arbeitstechniken, wie sie an einzelnen Arbeitsplätzen benötigt werden, Bestandteile des Assessments oder werden primär schulische Aspekte erfasst? Über diese Unterscheidungen hinweg hat sich eine Diskussion entfacht, die nach den Modellen beruflicher Kompetenz und vor allem nach den Inhalten und Methoden ihrer Messung fragt. Diese Diskussionen sind jedoch nicht immer ergebnisorientiert und münden deshalb noch kaum in nutzbaren Verfahren der Erfassung von Lernergebnissen oder in Evaluationsprogrammen.

\section{Zum Umgang mit Kompetenzmessungen und deren Ergebnissen}

Das Nutzen von empirischen Methoden zur Beschreibung von Ausbildungsprogrammen und deren Outcome sollte nicht nur als internationale Notwendigkeit, sondern vielmehr als Chance auf nationaler Ebene begriffen werden: für die Lehr-Lernforschung, die Fachdidaktiken, die Gestaltung von Aus- und Weiterbildungsprogrammen, die Lehrerbildung. Empirische Befunde sind jedoch nur dann legitimierbar, wenn sie die dem Kompetenzerwerb zu Grunde liegenden Lehr-Lernprozesse einschließlich der strukturellen Bedingungen in Schulen und Betrieben sowie den Fragen der didaktischen Ausgestaltung kompetenzorientierter Instruktion Rechnung tragen. Kompetenzforschung und die hier erzielten Evidenzen können dann durchaus - und dies ist ein wesentlich praktischer Beitrag - Motor für didaktische Innovation und Gestaltung sein. Um dies zu erreichen, ist die Kompetenzforschung auf drei Säulen zu stellen: 1) die Entmystifizierung des Konvolutes Kompetenz, 2) die Einführung der Curriculum-Instruktion-Assessment Triade als fachdidaktisches Leitbild sowie 3) die Erstellung von Handreichungen für kompetenzorientierte Aufgabenkonstruktionen für konkrete Unterrichts- und Arbeitssituationen bereits in der Lehrerausbildung. Über diese drei Säulen wird die Überzeugung transportiert, dass Kompetenzmessungen und deren Befunde vornehmlich der Verbesserung von Lern- und Arbeitsprozessen dienen sollen. Verfahren der Kompetenzmessung sollten daher so aufbereitet sein, dass sie - vor allem als Bestandteil von komplexen Lehr-Lern- 
Arrangements - curricular abgesichert als instruktionale Vorgaben dienen können; dies gilt gleichermaßen für die Rückmeldung der Befunde (u. a. Winther 2010; Achtenhagen und Winther 2011).

Diese Forderungen lassen sich aus den Erkenntnissen der internationalen Vergleichsstudien ableiten: Vorrangig bildungsökonomische Befunde (vgl. Wößmann 2007; Wößmann 2009) deuten u. a. darauf hin, dass Unterschiede in den Kompetenzausprägungen sich nicht ausschließlich auf die individuellen Dispositionen der Lernenden zurückführen lassen, sondern gleichsam auch durch institutionell bedingte Unterschiede im Umgang mit den curricularen Vorgaben oder unterschiedliche Instruktionskulturen (Institutionseffekte) veranlasst werden. Neben den Institutionseffekten werden zudem solche Effekte bedeutsam, die auf eine Assessment- bzw. Standardkultur abzielen. Hierfür sprechen auch Befunde, die zeigen, dass Probanden aus Ländern, in denen Evaluationsstandards erfolgreich implementiert wurden, bei Kompetenztests signifikant besser abschneiden. Mit diesen Beispielen soll nicht dafür plädiert werden, die Unterrichtspraxis statt an den Standards stärker an den Testsystemen auszurichten oder die Testkultur zum Steuerungsmittel zu erklären. Sie sollen jedoch dahingehend sensibilisieren, dass die Beschreibung und Messung individueller Kompetenzen auch immer an einen Kulturaspekt gebunden ist, den es in der Berufsbildungsforschung nachhaltig zu etablieren gilt. Oder anders formuliert: Die umfangreichen methodologischen Ausarbeitungen zur beruflichen Handlungskompetenz, zur Handlungsregulation in Arbeitsprozessen sowie zur Analyse von Lern- und Arbeitshandlungen, wie sie eine lange Tradition in der beruflichen Bildung haben, sind zu ergänzen um empirische Verfahren, die eine Beschreibung beruflichen Lernens und Arbeitens und damit beruflicher Professionalisierung ermöglichen.

Das Etablieren einer Testkultur ist u. E. eng an Accountability-Konzeptionen zu binden, vor deren Hintergrund Befunde beruflicher Assessments interpretierbar und vergleichbar werden (vgl. u. a. Herman 2008; Winther 2011). Mit Blick auf berufliche Kompetenzfeststellung sind insbesondere empirische Validierungen möglicher Testverfahren notwendig und überfällig. Dies gilt umso mehr, da die Befunde Ausstrahlungskraft besitzen können, die alle Systemebenen der Bildung und der Bildungsgestaltung berühren: auf der Makroebene als Steuerungshilfe, auf der Mesoebene als Gestaltungspotential für Institutionen und auf der Mikroebene als Umsetzungsparadigma für curriculare Reformen.

Gerade für die Messung beruflicher Kompetenzen wird der Anspruch erhoben, dass über die erzielten Befunde politisches Steuerungswissen ableitbar wird. In der beruflichen Bildung wird vor diesem Hintergrund vor allem folgende Frage zentral: Wie lässt sich insbesondere mit Blick auf die internationale Reputation des dualen Systems der Berufsausbildung ein allgemein akzeptiertes Assessment vorlegen, das im Kern auf die Erfassung beruflicher Handlungsfähigkeiten an realen Arbeitsplätzen abzielt? Die aktuellen Forschungsarbeiten im Feld der Berufsbildung versuchen hier insbesondere auf zwei Wegen Antworten zu finden: Es wird 1) eine stringente Verbindung zwischen fachdidaktischen und messtheoretischen Modellen angestrebt. Hierbei werden für eine kompetenzorientierte Instruktions- und Prüfungspraxis Implikationen deutlich, die direkt auf Mikro- und Mesoebene nutzbar sind. Die Studien sind 2) darauf hin ausgerichtet, deskriptive Aussagen zu personalen und institutionellen Bedingungen des Kompetenzerwerb zu generieren. Systemisch ausgerichtete nationale Projekte zur Beschreibung von Ausbildungsbedingungen und des Reformbedarfs in den Berufsbildungssystemen sind 
hier u. E. noch zu ergänzen. Weitere Forschungsdesiderate bestehen daneben vorrangig in der Ausarbeitung von Kommunikations-, Evaluations- und Umsetzungsstrategien im Hinblick auf neue Ansätze der Policy Transfer-Forschung.

U. E. sind damit Wege eingeschlagen, die den generellen Herausforderungen der Messung und (politischen) Bewertung von beruflichen Kompetenzen umfangreich Rechnung tragen. Dies gilt insbesondere dann, wenn Kompetenzmessungen den Anspruch verfolgen, aussagekräftig im Hinblick auf die Setzung eines normativen Referenzsystems für Leistungsfähigkeiten und für den Leistungsvergleich zu sein und gleichsam die aus den High-Stakes Testings bekannten Kritiken zu vermeiden. Legitimierbare Kompetenzmessverfahren und -befunde sollten daher Vorhersagekraft für zukünftige Entwicklungen besitzen, sie sollten keine Abhängigkeiten von den Curricula und den Lernformen aufweisen und eine rationale Anwendbarkeit und Verwertbarkeit ermöglichen (vgl. Berliner 2009a, 2009b).

\section{Inhalte und Besonderheiten dieses Heftes}

Im Rahmen dieses Heftes werden zwei Perspektiven der empirischen Berufsbildungsforschung aufgezeigt: Die Beschreibung von Ansätzen beruflicher Kompetenzmessungen einerseits sowie korrespondierend dazu andererseits Ansätze beruflicher Kompetenzentwicklungen, die vorrangig die Professionalisierung des Bildungspersonals in den Blick nehmen.

Die Perspektive I: „Berufliche Kompetenzentwicklung und Kompetenzmessung in den Berufen ", zeigt am Beispiel kaufmännischer, medizinischer, gewerblich-technischer sowie vollzeitschulischer Ausbildungsprogramme aktuelle Entwicklungen in der beruflichen Bildungsforschung auf. In allen Beiträgen wird deutlich, dass sich berufliche Kompetenzmessung durch zwei Facetten von der Kompetenzmessung im Bereich der allgemeinen Bildung unterscheiden lässt: 1) Entsprechend der Zielsetzung beruflicher Ausbildungsprogramme werden Modelle benötigt, die neben einer kognitiv akzentuierten Kompetenzauffassung auch die Fähigkeit zur Handlungsausführung berücksichtigen, um die Komplexität beruflicher Situationen adäquat abzubilden. In den Testkonstruktionen findet dies dadurch Berücksichtigung, dass berufliche Kompetenzen am Beispiel typischer Arbeitsprodukte sowie auf Basis authentischer Arbeitsaufgaben modelliert werden. Für den gewerblich-technischen und den kaufmännischen Bereich liegen hierfür entsprechende Beispiele vor. Die Besonderheiten der beruflichen Kompetenzmessung und -modellierung zeigen sich 2) darüber hinaus darin, dass strukturelle Zusammenhänge zwischen allgemeinen Grundqualifikationen und berufsspezifischen Kompetenzen aufzuklären sind, um beruflichen Kompetenzerwerb angemessen empirisch beschreiben zu können. Hiermit wird auch ein zentrales bildungspolitisches Problem berührt: Eine „Über“ betonung der Bedeutung basaler Fähigkeiten (Rechnen, Lesen, Schreiben) steht der strukturpolitischen Bewertung gegenüber, dass mit der zunehmenden Einbindung der beruflichen Bildung in das Berechtigungssystem der Stellenwert allgemeinbildender Fächer stärker stieg als der der beruflichen Inhalte (vgl. auch die aktuelle Debatte über die Einordnung beruflicher Bildungsabschlüsse in den Deutschen Qualifikationsrahmen (DQR)). Insgesamt greift die Perspektive der beruflichen Kompetenzmessung neben 
methodischen auch curriculare Aspekte auf und verdeutlicht damit die Notwendigkeit einer simultanen fachdidaktischen und messtheoretischen Aufbereitung beruflicher Testverfahren. Nur vor diesem Hintergrund können empirische Evidenzen über die Struktur und die Graduierung beruflicher Kompetenzen von Auszubildenden ein entsprechendes fachdidaktisches Potential entwickeln, das in Weiterentwicklungen der schulischen und betrieblichen Lehr-Lernprozesse einschließlich der Assessment- und Prüfungskultur einfließen kann.

Der einleitende Beitrag von Esther Winther und Viola Klotz greift Grundsatzfragen beruflicher Diagnostik unter dem Titel „Spezifika der beruflichen Kompetenzdiagnostik - Inhalte und Methodologie“" auf. In Form eines Problemaufrisses werden zunächst die Besonderheiten beruflicher Kompetenzmessungen sowie deren methodologische Implikationen elaboriert und anschließend exemplarisch vor dem Hintergrund ausgewählter Studien zur beruflichen Kompetenzmessung in der kaufmännischen Domäne beschrieben. Der Beitrag zeigt insbesondere die Notwendigkeit einer fachdidaktischen und messtheoretischen Interaktion in den Kompetenztestverfahren auf und plädiert für eine größere Offenheit gegenüber quantitativ empirischen Methoden in der Berufsbildungsforschung.

Eine notwendig fachdidaktische Auseinandersetzung im Rahmen beruflicher Kompetenzmessungen wird auch von Susanne Weber und Frank Achtenhagen hervorgehoben. In ihrem Beitrag „Fachdidaktisch gesteuerte Modellierung und Messung von Kompetenzen im Bereich der beruflichen Bildung" wird beispielhaft für die Ausbildung im Bereich Wirtschaft und Verwaltung ein Vorschlag zur Modellierung und Messung interkultureller Kompetenz entworfen. In Weiterführung eines als Design-Experiment angelegten Projekts zur Förderung interkulturellen Lernens wird ein auf fachdidaktischen Überlegungen basierendes Kompetenzstufenmodell vorgestellt und auf seine empirische Belastbarkeit hin überprüft. Die statistischen Auswertungen belegen, dass sich das theoretische Modell der angenommenen Graduierung interkultureller Kompetenz bestätigen lässt - dies erlaubt detaillierte Analysen, mit deren Hilfe sich das Niveau der erworbenen interkulturellen Kompetenz individuell ermitteln lässt; zugleich können Aufgaben mit entsprechenden Schwierigkeitsabstufungen formuliert werden.

Im Beitrag „Struktur und kognitive Voraussetzungen beruflicher Fachkompetenz: Am Beispiel Medizinischer und Zahnmedizinischer Fachangestellter" von Susan Seeber werden unerwartete empirische Befunde zum Anlass genommen, die Modellierung beruflicher Fachkompetenz in den Kontext allgemeiner kognitiver Ressourcen und Basiskompetenzen zu stellen. Die Ergebnisse belegen einerseits starke Zusammenhänge zwischen Basiskompetenzen und beruflichen Fachleistungen für die beiden untersuchten medizinischen Berufe und verweisen andererseits auf Unterschiede in den Fachleistungen, die sich auf Heterogenitätseffekte zurückführen lassen.

Der Bedeutung von Basiskompetenzen für die Entwicklung fachspezifischer Kompetenzen wird auch im Beitrag von Cordula Petsch, Kerstin Norwig und Reinhold Nickolaus nachgegangen. Am Beispiel gewerblich-technischer Ausbildungsgänge werden Ansätze der „Kompetenzförderung leistungsschwächerer Jugendlicher in der beruflichen Bildung" vorgestellt. Zentral ist dabei die Frage, inwieweit in der beruflichen Bildung mit einer gezielten Förderung der Basiskompetenzen bestehende Entwicklungsbarrieren reduziert werden können. Mittels zweier Interventionsstudien wird das Förderkonzept „BEST“ beschrieben und evaluiert. Die Besonderheit dieses Förderkonzepts zeigt sich 
darin, dass sowohl die kognitiv starken als auch die kognitiv schwächeren Jugendlichen von der Förderung profitieren und positive Kompetenzentwicklungen angestoßen werden können.

Stephan Schumann und Franz Eberle setzen sich in ihrem Forschungsbeitrag „Ökonomische Kompetenzen von Lernenden am Ende der Sekundarstufe II“" mit der Struktur und der Ausprägung ökonomischer Kompetenzen von Lernenden in vollzeitschulischen Bildungsgängen in der Schweiz auseinander. Die Grundlage bildet ein komplexes Modell, in das neben ökonomischem Wissen und Können insbesondere auch wirtschaftsbezogene motivationale Orientierungen sowie Einstellungen und Werthaltungen zu wirtschaftlichen Problemstellungen einfließen. Die Befunde verweisen auf ein valides und reliables Testinstrumentarium, mit dem es gelingt, die Ausprägung sowie ausgewählte Korrelate ökonomischer Kompetenzen zu beschreiben und in Erklärungszusammenhänge zu stellen.

Die Perspektive II: „Professionalisierung des Bildungspersonals “, nimmt Studien in den Blick, die Fragen der Aus- und Weiterbildung von Lehrkräften und Ausbildern in beruflichen Domänen in den aktuellen Kompetenzdiskurs einbinden. Es liegen bislang kaum empirische Arbeiten aus der beruflichen Bildung vor, die die strukturellen Beziehungen zwischen allgemeinen und fachspezifischen Motivationsfacetten, epistemologischen Überzeugungen, fachwissenschaftlichem und fachdidaktischem Wissen sowie dem professionellem Selbstverständnis quantitativ-empirisch beschreiben. Aktuelle Studien greifen dieses Forschungsdesiderat auf und begünstigen durch ihren methodischen Zugang eine Neuausrichtung der theoretischen Perspektive der Lehrprofessionalität abseits von generalisierten Erziehungserwartungen (vgl. hierzu die Beiträge in Zlatkin-Troitschanskaia, et al. 2009). Ein zentraler Befund dieser Studien ist es, dass Untersuchungen zur Lehrprofessionalität domänenspezifisch anzulegen sind. Dies ist umso bedeutsamer, wenn berücksichtigt wird, dass der Kompetenzerwerb und die Kompetenzentwicklung von Auszubildenden im beruflichen Bereich nicht nur von den Schulen, sondern zu einem großen Teil von den Ausbildungsleistungen der Betriebe getragen werden.

Die Ausbildungsleistungen der Betriebe stehen im Fokus des Beitrags „Ausbleibende Effekte pädagogischer Professionalisierung des betrieblichen Ausbildungspersonals: Ergebnisse einer Längsschnittstudie" der Autoren Andreas Rausch, Jürgen Seifried und Christian Harteis. Ziel der Studie ist es, den Einfluss pädagogischer Weiterbildungsmaßnahmen auf die Ausbildungsqualität empirisch zu beschreiben. Hierbei werden neben Betreuungsauffassungen insbesondere verschiedene pädagogische Sichtweisen in den Blick genommen, um die Frage zu beantworten, wie sich Maßnahmen der pädagogischen Professionalisierung des Ausbildungspersonals auf die Betreuungs- und Ausbildungsqualität und damit auch auf die Kompetenzentwicklung der Auszubildenden auswirken.

Christiane Kuhn, Roland Happ, Olga Zlatkin-Troitschanskaia, Klaus Beck, Manuel Förster und Daja Preuße befassen sich hingegen mit der „Kompetenzentwicklung angehender Lehrkräfte im kaufmännisch-verwaltenden Bereich - Erfassung und Zusammenhänge von Fachwissen und fachdidaktischem Wissen“. Kernstück des Beitrags stellt die Validierung eines Tests zum fachdidaktischen Wissen dar, mit dessen Hilfe der Zusammenhang zwischen fachdidaktischer und fachwissenschaftlicher Kompetenzentwicklung aufgeklärt werden kann. Mit diesem methodischen Zugang liefert der Beitrag erste empirisch fundierte Hinweise zur spezifischen Struktur der professionellen Kompetenz von (angehenden) Lehrkräften im kaufmännisch-verwaltenden Bereich. 


\section{Literatur}

Achtenhagen, F., \& Winther, E. (2011). Fachdidaktische Perspektiven der Kompetenzmessung am Beispiel des kaufmännisch-verwaltenden Bereichs. In O. Zlatkin-Troitschanskaia (Hrsg.), Stationen Empirischer Bildungsforschung. Traditionslinien und Perspektiven (S. 352-367). Hohengehren: Schneider.

Baethge, M., Achtenhagen, F., Arends, L., Babic, E., Baethge-Kinsky, V., \& Weber, S. (2006). Berufsbildungs-PISA-Machbarkeitsstudie. Stuttgart.

Berliner, D. C. (2009a). Do High-stakes tests of academic achievement limit economic development? The beginning of a long-term natural longitudinal study. In F. Oser, U. Renold, E. R. John, E. Winther, \& S. Weber (Hrsg.), VET Boost: Towards a theory of professional competencies. Essays in honor of Frank Achtenhagen (S. 55-70). Rotterdam: Sense publishers.

Berliner, D. C. (2009b). The incompatibility of high-stakes testing and the development of skills for the 21st century. In R. Marzanno (Hrsg.), On excellence in teaching, 2009. Bloomington: Solution Tree Press.

Herman, J. L. (2008). Accountability and assessment: Is public interest in K-12 education being served? In K. E. Ryan \& L. A. Shepard (Hrsg.), The Future of Test-Based Educational Accountability (S. 211-232). New York: Routledge.

Ryan E., \& Shepard L. A. (2008) (Hrsg.). The future of test-based educational accountability (S. 211-232). New York: Routledge.

Seeber, S., Nickolaus, R., Winther, E., Achtenhagen, F., Breuer, K., et al. (2010). Kompetenzdiagnostik in der Berufsbildung Begründung und Ausgestaltung eines Forschungsprogramms. Berufsbildung in Wissenschaft und Praxis, Beilage zu 1/2010, 1-15.

Walker, D. A., Anderson, C. A., \& Wolf, R. M. (1976). The IEA six subject survey: An empirical study of education in twenty-one countries. New York: Almqvist \& Wiksell.

Wilson, M. (2004). Assessment, accountability and the classroom: A community of judgement. In M. Wilson (Hrsg.), Towards coherences between classroom assessment and accountability. 103rd yearbook of the national society for the study of education (S. 1-19). Chicago: The University of Chicago Press.

Winther, E. (2010). Kompetenzmessung in der beruflichen Bildung. Bielefeld: Bertelsmann.

Winther, E. (2011). Das ist doch nicht fair! - Mehrdimensionalität und Testfairness in kaufmännischen Assessments. Zeitschrift für Berufs- und Wirtschaftspädagogik, 107(2), 218-238.

Wolf, A. (1995). Competence-based assessment. Oxfordshire: Marston Lindsay Ross.

Wößmann, L. (2007). International evidence on school competition, autonomy and accountability: A review. Peabody Journal of Education, 82(2-3), 473-497.

Wößmann, L. (2009). Bildungssystem, PISA-Leistungen und volkswirtschaftliches Wachstum. Ifo, 62(10), 23-28.

Zlatkin-Troitschanskaia, O., Beck, K., Sembill, D., Nickolaus, R., \& Mulder, R. (Hrsg.). (2009). Lehr-professionalität. Bedingungen, Genese, Wirkungen und ihre Messung. Weinheim: Beltz. 